\title{
EchoGéo
}

44 | 2018

Les valorisations territoriales et touristiques du street art

\section{Les Magasins généraux : de spot à vandales à spot publicitaire?}

\section{Constance Ananos}

\section{OpenEdition}

\section{Journals}

Édition électronique

URL : https://journals.openedition.org/echogeo/15673

DOI : 10.4000/echogeo. 15673

ISSN : 1963-1197

Éditeur

Pôle de recherche pour l'organisation et la diffusion de l'information géographique (CNRS UMR 8586)

Référence électronique

Constance Ananos, "Les Magasins généraux : de spot à vandales à spot publicitaire? ", EchoGéo [En ligne], 44 | 2018, mis en ligne le 31 juillet 2018, consulté le 01 août 2021. URL : http://

journals.openedition.org/echogeo/15673; DOI : https://doi.org/10.4000/echogeo.15673

Ce document a été généré automatiquement le 1 août 2021

EchoGéo est mis à disposition selon les termes de la licence Creative Commons Attribution - Pas d'Utilisation Commerciale - Pas de Modification 4.0 International (CC BY-NC-ND) 


\section{Les Magasins généraux : de spot à vandales à spot publicitaire?}

\section{Constance Ananos}

Illustration 1 - Le bâtiment des douanes en 2011

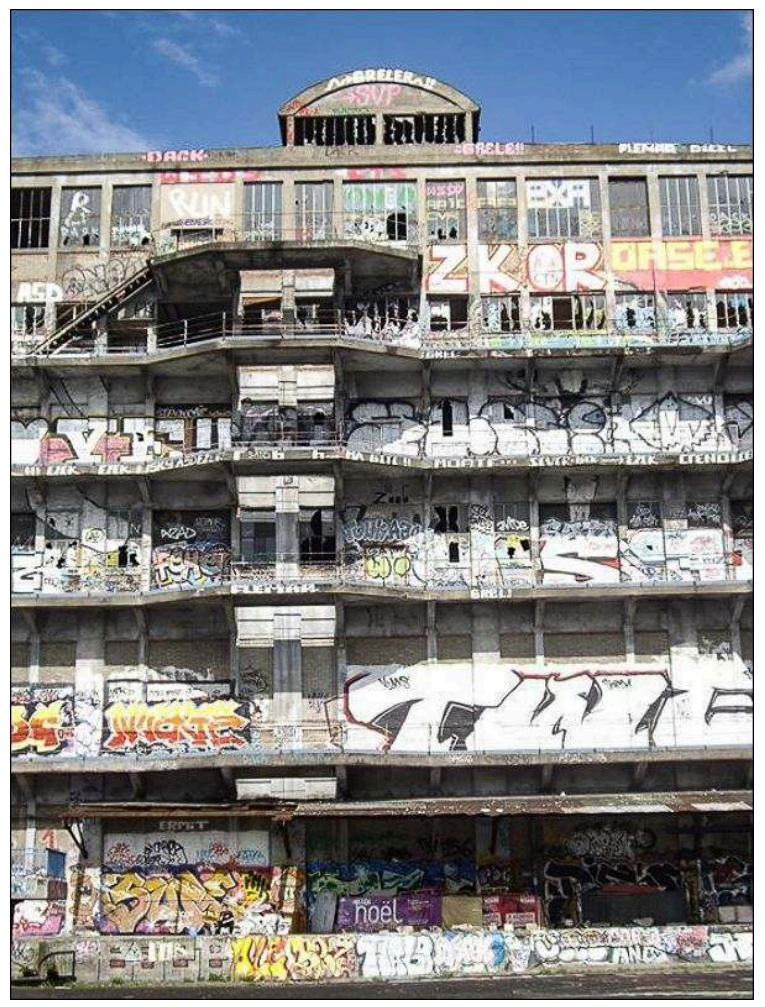

Auteur : C. Ananos

Cet article s'intéresse aux pratiques du graffiti et du street art en s'appuyant sur la réhabilitation du bâtiment des douanes à Pantin (Seine-Saint-Denis), commune limitrophe de la capitale. 
2 Dans les sciences humaines et sociales, le graffiti et le street art sont des objets de recherche délicats à approcher, du fait des ambiguïtés et contradictions soulevées par la multiplicité de leurs champs et modes d'action. Ils font l'objet d'une littérature florissante, sous forme d'études locales commandées par les grandes villes françaises ou de publications scientifiques dans de nombreuses disciplines : que ce soit en histoire de l'art et arts plastiques, en sociologie, sur les profils et revendications des graffeurs (Kokkoref, 1988) et en sciences politiques, sur les graffitis new-yorkais (Baudrillard, 1975) et plus récemment sur l'effacement des graffitis (Vaslin, 2017). Un des ouvrages les plus conséquents est issu des études esthétiques et culturelles (Génin, 2016) mais nous retrouvons les questions de propriété intellectuelle et droit d'auteur (Gré, 2014) posées dans le domaine juridique ou bien la question du phénomène de gentrification (Blanchard, 2017) en géographie urbaine. Le graffiti et le street art ont aussi fait l'objet de nombreux articles journalistiques et livres au-delà de la recherche universitaire, souvent dans un but de valorisation ou de critique de ces pratiques et/ou des opérations urbaines les impliquant.

3 Cette étude de cas portant sur les Magasins généraux choisit une approche socioéconomique et politique pour comprendre le rôle des différents acteurs de la scène graffiti et street art dans les processus d'institutionnalisation et de patrimonialisation de ces pratiques urbaines. La rénovation du bâtiment des anciennes douanes de Pantin s'inscrit dans le cadre de la mise en place d'une Zone d'aménagement concertée (ZAC) centrée sur le Port de Pantin. Cette ZAC a pour objectif de créer une nouvelle centralité en faisant émerger un quartier sur l'axe du canal de l'Ourcq (qui scinde la commune en deux). Ces projets font partie d'une stratégie globale de transition économique et rénovation urbaine sur le territoire communal dans son ensemble. Cette recherche part du principe d'une reconnaissance et d'une valorisation accrue de l'art dans l'espace public (Guinard, 2014), et prend acte d'une reconnaissance et institutionnalisation du graffiti et du street art dans le contexte des processus de production artistique in et off (Vivant, 2007) et de la ville créative (Vivant, 2009). Il s'agit en particulier de s'intéresser à un acteur original de cette opération d'envergure, BETC Paris (du groupe mondial HAVAS), une agence publicitaire française qui est actuellement le gestionnaire du lieu public des Magasins généraux. Dans un contexte de gentrification du Nord-Est parisien et de Paris, et de l'axe du canal de l'Ourcq à Pantin, déjà mis en évidence par nombre d'auteurs ${ }^{1}$, il est en effet légitime de se demander pourquoi et comment une entreprise privée dont le champ de compétences est la publicité en est venue à devenir un acteur majeur de la fabrique urbaine culturelle sur un territoire stratégique du fait de sa proximité spatiale et économique avec la capitale française et de sa participation au projet du grand Paris, mais aussi du fait d'un processus de redéfinition des économies locales ayant déjà attiré d'autres acteurs conséquents du paysage économique, par exemple BNP Paribas, Hermès ou la galerie d'art Thaddeus Ropac.

4 La recherche sur les Magasins généraux se base sur différentes méthodes de recherche allant de la consultation d'ouvrages et articles, avec une attention particulière portée aux productions journalistiques sur le graffiti et le street art et aux médias locaux, à l'enquête de terrain de type ethnographique qui s'appuie sur des observations participatives dans les milieux du graffiti et du street art, des entretiens formels avec des fonctionnaires des pouvoirs publics territoriaux ${ }^{2}$ et informels avec les artistes (graffeurs et street artistes) mais aussi la consultation et le suivi des profils Facebook (principalement) des acteurs concernés. À cela s'ajoutent une expérience personnelle 
de fréquentation régulière de la commune de Pantin et du lieu de 2010 à 2013, la participation à certains événements culturels organisés par les Magasins généraux entre 2016 et 2017, ainsi qu'une expérience professionnelle de médiatrice culturelle au sein du département de la culture à la mairie de Pantin de septembre 2011 à juin 2012.

\section{Histoire de défricher le terrain}

\section{Les Magasins généraux, le graffiti et le street art : des rencontres prédéterminées?}

Illustration 2 - Éléments du bâtiment des douanes en 2012

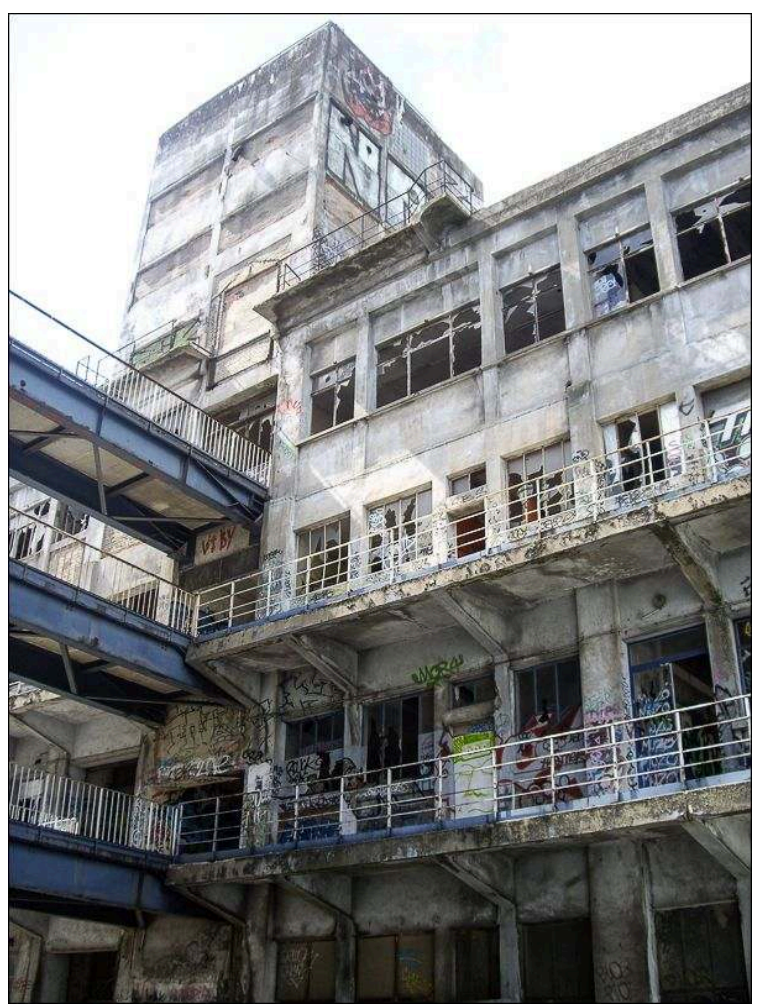

Auteur : C. Ananos

Les Magasins généraux constituent un grand bâtiment scindé en deux structures de béton armé reliées par des passerelles. Initialement conçus comme entrepôts de marchandises par la Chambre de Commerce de Paris (CCP) à la fin des années 1920, ils sont mis en service en 1931. Après 70 ans d'activité, ils ferment leurs portes en 2001, à la suite de l'élection du candidat socialiste Bertrand Kern, qui ne s'accorde pas avec les projets de la Chambre de Commerce de Paris quant à l'avenir du lieu. En effet, en vue de progressivement réduire la présence automobile dans le centre pantinois, le nouveau maire pantinois s'oppose au projet de la CCP qui souhaite développer un centre routier pour camions de façon à rentabiliser le bâtiment (Rigaud, 2015). En 2004, la Société d'économie mixte de Pantin (Semip) rachète les terrains des Magasins généraux. La question du devenir du bâtiment se pose alors que celui-ci est vidé de son activité industrielle et devient une friche urbaine. En 2006, la municipalité lance le projet d'un nouveau quartier autour du canal de l'Ourcq avec la création de la ZAC du Port de 
Pantin de 6,5 hectares. Le territoire de la commune de Pantin est historiquement morcelé ${ }^{3}$ et ce projet sous-tend une stratégie de faire émerger une centralité urbaine sur l'axe de ce nouveau quartier du canal.

Alors que cette réflexion de future réhabilitation se met doucement en place, le bâtiment, alors délaissé, est peu à peu investi par des graffeurs et des street artistes. Soulignons qu'en France, le graffiti et le street art se distinguent l'un de l'autre historiquement et techniquement. Le graffiti, tel que considéré ici, provient des ÉtatsUnis. Il se caractérise par un sujet artistique autour de la lettre et du lettrage et une volonté de s'approprier illégalement des morceaux de paysage urbain, des spots selon la terminologie que les pratiquants emploient. Le graffiti répond à un ensemble de codes esthétiques et de règles sociales qui se régulent de façon autonome. Entre artisans de la lettre et artistes du lettrage, les graffeurs intègrent le tag, le flop ${ }^{4}$, la pièce ${ }^{5}$, mais aussi des personnages et des éléments figuratifs tels que les étoiles, les flèches, les couronnes, les nuages.

7 L'art de rue en soi n'est pas un phénomène nouveau à l'arrivée du graffiti en Europe dans les années 1980, le terme graffiti était et est toujours employé pour désigner les écritures informelles des lieux publics comme l'a montré le travail de recherche du photographe Brassaï sur les graffitis parisiens (1961).

8 À Pantin, le bâtiment des douanes est un parfait exemple des différentes approches du graffiti car, comme il est visible dans les illustrations, il regroupe des tags, des flops, des lettrages des personnages, des pièces individuelles, d'autres collectives; des pièces mettant en valeur le blase, le pseudonyme, du graffeur, d'autres celui du crew ${ }^{6}$. La figure 1, montrant la façade du bâtiment avant la commande artistique auprès du collectif PoDaMa dans la cadre de l'Eté du canal, illustre ces différents styles de lettrage dans le graffiti : au rez-de-chaussée et troisième étage, on distingue des flops en chrome et noir ; au premier on voit l'imposant TWE qui a été conservé lors de l'intervention de PoDaMa en 2012, à la demande du crew, et sur ordre du Comité départementale de tourisme 93 qui a accédé à leur demande. Ci-dessous, la figure trois représente une œuvre collective des TWE, avec un lettrage "twe krew» et un personnage central, combinaison typique dans le graffiti ; on distingue dessous des flops et des tags. 


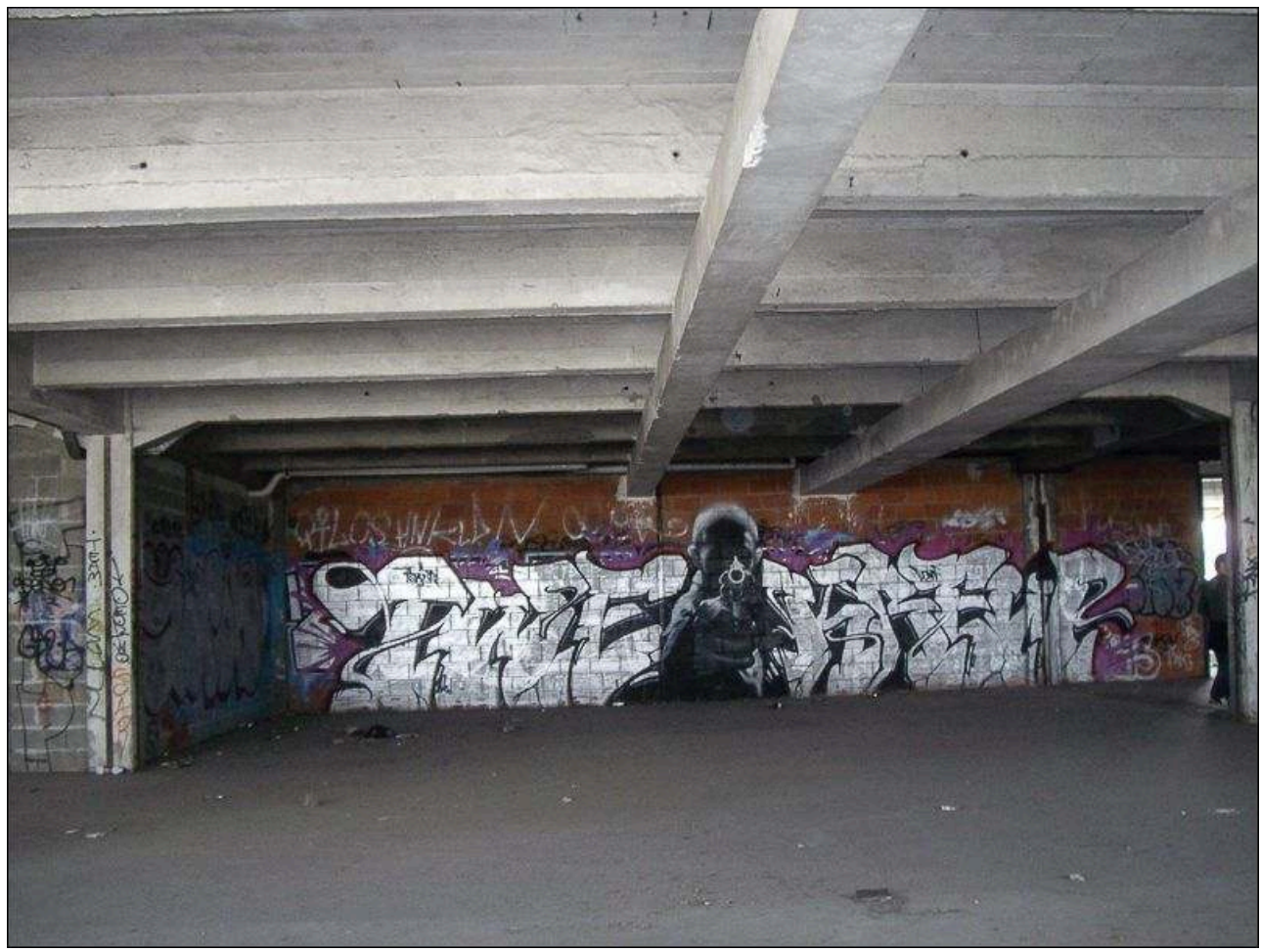

Lettrage par Skuz et personnage par Flow réalisés en 2010.

Auteur: C. Ananos

Le street art est aussi présent dans et sur le bâtiment, bien que sa présence soit moins marquée en comparaison avec les nombreuses pièces de graffiti. On y trouve cependant des œuvres esthétiquement distinctes du graffiti et dont les techniques sont assimilées au street art, des pochoirs par exemple. Le street art, distinct de sa traduction littérale française "art de rue ", émerge dès les années 1960 et 1970. Les deux pratiques se croisent historiquement, parfois esthétiquement, leurs frontières symboliques sont poreuses. Il s'avère qu'une friche à caractère industriel est typiquement le genre de lieu où le graffiti et le street art vont cohabiter voire collaborer.

\section{Émergence d'un lieu off avec la friche urbaine du « bâtiment des douanes ", future " cathédrale du graff »}

Dans le contexte de la ville contemporaine, le graffiti et le street art correspondent à la définition de pratiques off donnée par Elsa Vivant (2007) : elles sont innovantes à la fois par leurs façons d'appréhender les espaces urbains et par les supports artistiques utilisés, et par leur interaction indirecte ou directe avec le passant et/ou spectateur et surtout parce qu'elles amènent à appréhender les espaces collectifs (publics) différemment et à questionner leur composition. Elles peuvent à la fois être destinées à un public qui saura où aller les chercher, notamment pour le graffiti illégal, mais aussi à un public plus large et varié du fait de la diffusion des pièces sur internet (réseaux sociaux et sites thématiques) par le biais de la photographie et de la vidéo ; elles sont aussi légales ou illégales en fonction du contexte de production. Elles sont aujourd'hui un marqueur symbolique constitutif de nombreux milieux urbains occidentaux et sont associées aux notions d'art, de créativité, et de marginalité. Elles participent à la 
production d'événements off, des événements conçus et produits en dehors des sphères institutionnelles et commerciales et «[...] utilisent de manière plus ou moins temporaire des lieux divers qui sont à la fois des lieux off de la culture et lieux de la culture off » (Vivant, 2007).

11 Au début des années 2000 avec le développement du graffiti et du street art, leur succès médiatique et les processus de transformation urbaine, les lieux et espaces propices au graffiti se sont fait plus rares, notamment car ils ont été de plus en plus fréquentés, donc peints, par les différents artistes interagissant avec la rue. En parallèle, des graffeurs de terrain, qualifiés «de terrain » justement parce qu'ils appréhendent le graffiti avec une importance moindre accordée aux risques pris et à la compétition du blase comparée l'attention portée à l'élaboration artistique en tant que telle, cherchaient de nouveaux lieux à investir sans cette fréquentation assidue des acteurs du graffiti et du street art.

Illustration 4 - Lek et Bisk

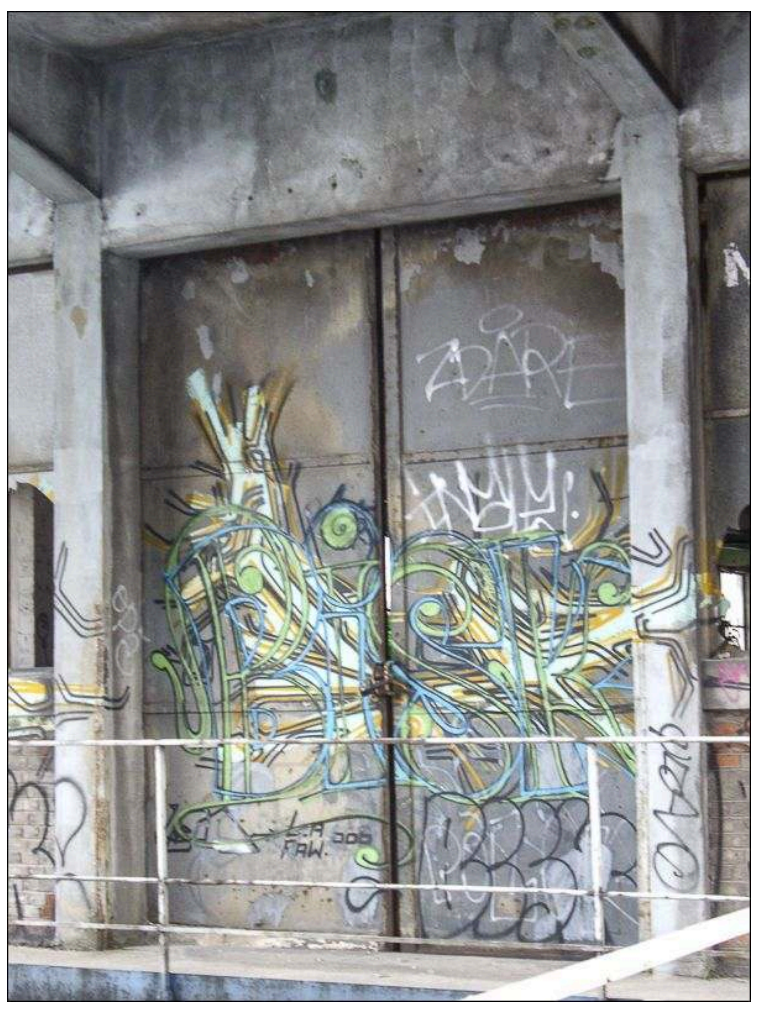

Un lettrage de Bisk s'est installé sur l'œuvre de Lek

Auteur: C. Ananos.

C'est pourquoi le bâtiment des douanes était déjà sous surveillance d'acteurs du off tels que le graffeur Lek, qui attendaient sa fermeture définitive pour aller l'explorer et le peindre. Les premiers à oser s'aventurer dans le bâtiment sont des graffeurs de passage, à partir de 2004, dont les pièces ont été diffusées dans les médias spécialisés, ce qui attirent d'autres graffeurs. Lek, lui, investit le lieu en 2006, il partage ce spot avec des compères, eux aussi graffeurs, Horfé, Fléo et Gorey ${ }^{7}$. Vient ensuite le crew de graffeurs, les TWE, qui va investir le lieu conséquemment de 2008 aux alentours de 2012.

13 Crew connu et reconnu du Nord parisien, fondé par le graffeur Arone ${ }^{8}$, les TWE vont peu à peu faire des Magasins généraux leur lieu de prédilection, une sorte d'atelier 
d'art, espace de loisir et de création artistique. C'est Arone qui découvre l'endroit en 2008 par l'intermédiaire de Veans; et tombe sous le charme du lieu : "J'aimais bien qu'on investisse un lieu entier comme on l'avait déjà fait déjà dans l'entrepôt de Saint-Ouen, par exemple. C'est une technique qui paie, car ça marque beaucoup plus les graffeurs qui entrent que peindre un petit peu dans plein de terrains différents" (Boukercha, 2014). De la même manière qu'Ypope était venu peindre le bâtiment après avoir vu les pièces de Tonka et Vinil circuler dans la presse spécialisée, Arone découvre les œuvres de Lek, Horfé et Gorey (etc.) ; s'en inspire autant que du bâtiment en soi qui l'a séduit par son caractère industriel. C'est un des modes de fonctionnement de ce crew que de trouver de grands espaces délaissés, avec de grands murs lisses, où ils ont la possibilité de développer leur panel de techniques et styles c'est pourquoi les anciens lieux industriels sont particulièrement attractifs. Il en résulte de nombreuses productions des TWE, sur les murs intérieurs comme sur les façades extérieures du bâtiment; des productions singulières et d'autres collectives. Les lieux restent difficiles d'accès et les graffeurs ne sont pas les seuls à trouver un intérêt au bâtiment : «Au début, il n'y avait pas encore de palissades, alors on entrait par les trous dans les fenêtres. [...] Par la suite, il y a eu un camp de travailleurs clandestins. [...] Après ça, sont arrivés les premiers baraquements de chantier avec un agent de sécurité, là pour surveiller'9. [...] Pour toutes ces raisons, la plupart des graffeurs qui venaient dans le bâtiment n'y revenaient pas quotidiennement comme nous » (Boukercha, 2014).

Les processus d'appropriation des graffeurs sur cette friche se sont faits par l'exploration et l'investissement social du lieu, Lek et ses compères l'ont transformé en terrain de jeu artistique avec des œuvres indépendantes et collectives, qui s'interpellent et se répondent dans le bâtiment. Lek indique avoir été inspiré par l'architecture du bâtiment, et son œuvre s'imbrique dans celle-ci. 


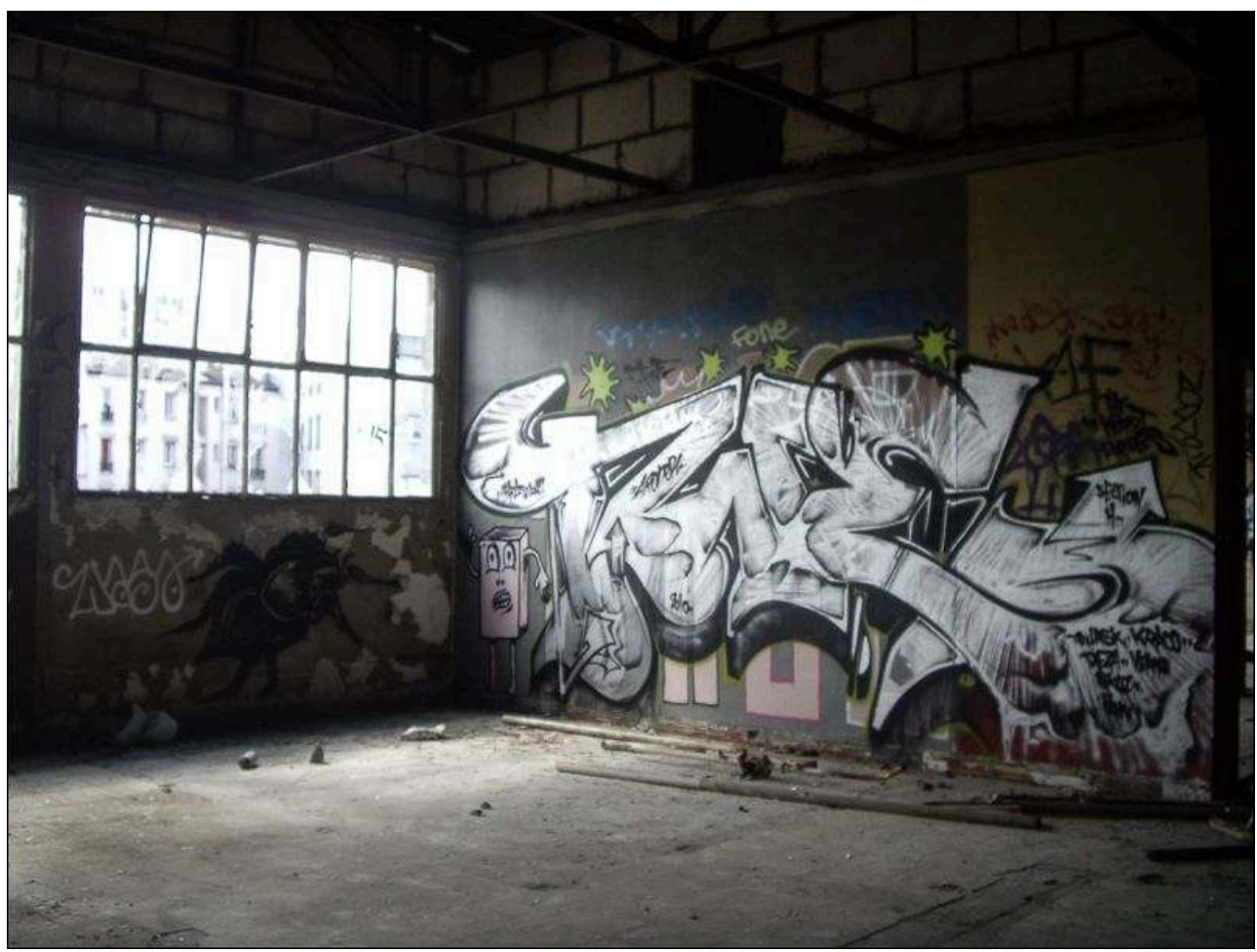

Un lettrage TWE par Arone et des insectes par Itvan Kebadian, deux noms pour un même artiste. Auteur: C. Ananos

Arone et les TWE ont fait du bâtiment un lieu de socialisation, où se retrouver et peindre ensemble, autant qu'un atelier d'art où affiner leurs techniques et en expérimenter d'autres. C'est le cas d'Itvan Kebadian, véritable nom d'Arone, qui s'est appliqué à parsemer ses insectes comme celui visible sur la figure 4 dans tout le bâtiment, transformant le bâtiment en nid pour ses peintures. Il a aussi réalisé des fragments de paysages urbains avec la même palette de couleur et la même technique de peinture.

Il est intéressant de constater que les acteurs du off sont ici principalement des graffeurs, l'occupation TWE en étant le meilleur exemple. Cependant les œuvres réalisées par Lek ou Itvan Kebadian sont esthétiquement plus proches du street art car elles se sont détachées de la primeur du lettrage. L'identité artistique multiple d'Arone / Itvan Kebadian met en évidence la différence entre les deux milieux autant que leurs liens. Les graffeurs ne sont d'ailleurs pas les seuls à avoir orné les murs du lieu de couleurs, il était aussi possible de voir des phrases du franco-écossais Sean Hart, qui s'attache plutôt à réaliser des jeux de mots et des interpellations philosophiques et/ou poétiques sur les murs sans revendiquer une affiliation au milieu graffiti.

17 Cette appropriation orchestrée par les artistes et pour les artistes off du lieu par l'exploration et l'expérimentation artistique a donné une valeur symbolique (Vivant, 2007) aux Magasins généraux qui se sont transformés en lieu off de l'art. Le bâtiment s'est retrouvé bardé de couleurs entre les tags, les flops, les pièces, les pochoirs et les peintures figuratives. Ces acteurs du off lui ont donné d'autres usages : celui de musée, d'atelier d'art, de lieu de sociabilisation. Ce faisant, ils ont créé des événements off par leur présence et leur pratique artistique: exploration urbaine par la visite du lieu, 
rassemblement amical, moment de peinture collective ou individuelle... Ils ont ainsi inscrit les Magasins généraux par ces transformations d'usages dans le contexte pantinois qui opère une transition post-industrielle. Ils ont contribué à en changer le paysage urbain en changeant l'esthétique du bâtiment, lui donnant ainsi cette valeur symbolique.

\section{Mise en événement et en scène d'un lieu off dans une stratégie touristique et patrimoniale}

18 La commune de Pantin connaît un processus de gentrification, au sens "forme particulière d'embourgeoisement qui transforme la composition sociale comme le bâti et l'espace public des quartiers populaires » (Clerval et Fleury, 2009), notamment sur l'axe du canal avec l'installation d'entreprises conséquentes et la construction de logement à destination d'une classe moyenne diplômée attirée par l'esthétique et le dynamisme culturel et économique d'un territoire (Vivant, 2009), bien que la mairie impose systématiquement $33 \%$ de logements sociaux dans les projets immobiliers sur son territoire.

Le projet d'aménagement de la zone du Port de Pantin implique une série de changements d'usages des espaces composant cette zone; le port qui n'avait plus vraiment de fonction depuis l'arrêt de l'activité industrielle devient un port de plaisance. Un groupe scolaire et des logements sont édifiés et de nouveaux espaces publics sont pensés pour bâtir un projet urbain qui correspond aux valeurs promues par la municipalité, en l'occurrence l'éducation, le logement, le loisir et la culture. Le bâtiment des douanes est un des piliers du projet : de par sa stature imposante et son histoire, il est un repère du paysage pantinois, un marqueur symbolique autant que physique de son histoire industrielle liée à la capitale. Réussir sa rénovation urbaine était donc un enjeu capital pour marquer la transition urbaine de la zone du Port et du canal. Il incarne ainsi le renouveau du tissu économique et culturel pantinois en y accueillant un des espaces publics de la ZAC du Port et en devenant le siège d'une agence publicitaire. Le graffiti et le street art sont ici les outils pour mettre en scène et en événement cette transition urbaine, la rénovation du lieu aussi bien que l'installation de cette agence, ils servent à ancrer symboliquement les opérations urbaines mises en place en donnant une légitimité artistique, un héritage culturel à revendiquer. Aussi, l'installation d'une agence dont le domaine de compétences est la publicité ${ }^{10}$ participe à la construction de l'image de Pantin en ville créative ; c'est là que les intérêts des différents acteurs convergent.

La commune de Pantin administre et gère le territoire via la Semip, outil de gestion immobilière et financière. Le comité départemental de tourisme de la Seine-Saint-Denis s'attèle à mener une politique de développement et de promotion touristique du territoire départemental, celui du «9-3». Soulignons l'engagement du directeur du CDT pour un tourisme durable et alternatif, ce qui a poussé le CDT à s'intéresser au graffiti et au street art. La municipalité et le CDT93 partagent le territoire communal comme territoire d'action et des objectifs communs de développement socioéconomique et promotionnel de ce territoire. Les Magasins généraux s'inscrivent dans leurs objectifs car il a l'étoffe pour s'imposer en centre culturel à rayonnement départemental et métropolitain, donc qui dépasse les frontières de la commune. En le mettant au centre d'une série d'opérations à but promotionnel et touristique, l'objectif 
est de changer l'image de la ville et du département tout en renouvelant le tissu économique local durement touché par les processus de désindustrialisation du NordEst parisien. Ces opérations ne sont pas isolées et s'inscrivent à la fois dans le contexte de la mise en place du grand Paris et dans celui de réhabilitation (et de gentrification) urbaine des territoires de l'Est parisien (Clerval et Fleury, 2009).

21 Dès 2011, le graffiti et le street art sont intégrés dans la série d'événements festifs programmés chaque année aux mois de juillet et d'août dans le cadre de l'Eté du Canal, organisé par le CDT93, par le biais de visites guidées et de balades urbaines sur la thématique du graffiti et du street art. Initialement localisées dans le $19^{\mathrm{e}}$ arrondissement de Paris et réalisées par l'artiste DaCruz, natif de cet arrondissement, ces visites se sont étendues jusqu'à Bobigny. Elles se sont aussi diversifiées entre la promenade à pied et celle en bateau sur le canal de l'Ourcq. Différents guides dont d'autres street artistes tels que Thom Thom se sont prêtés au jeu. En parallèle, DaCruz, Popof et Marko, qui forment le collectif PoDaMa, réalisent des performances publiques ensemble et séparément le long du canal (à Bondy, Pantin, Noisy-le-Sec, etc.). Des panneaux de bois sont installés au pied des Magasins généraux pour ce type de performance en 2012, à côté d'une péniche qui propose boissons et en-cas à bord. Pour l'été 2018, ce même type de balade est de nouveau proposé, avec DaCruz comme guide.

En 2012, le collectif PoDaMa, sous l'égide du CDT93, se voit confier un libre accès aux Magasins généraux, c'est le projet «Graff « pharaonique » [...] Renaissance du bâtiment des douanes de Pantin sous les graffs », comme le titre la programmation disponible sur le site web du CDT93, qui, par ailleurs, a désormais une section "Art urbain » sur sa page d'accueil, entre la section «Famille», et "Grand Paris ». Le collectif d'artistes invite d'autres artistes à les aider à peindre le bâtiment, notamment le graffeur brésilien Alexandre Orion et les français Dem189 et Julien Malland alias Seth. Après qu'ils aient terminé de peindre le lieu, le bâtiment a été mis en lumière lors des Journées du Patrimoine, en septembre 2012, et des visites payantes du bâtiment ont été organisées. 


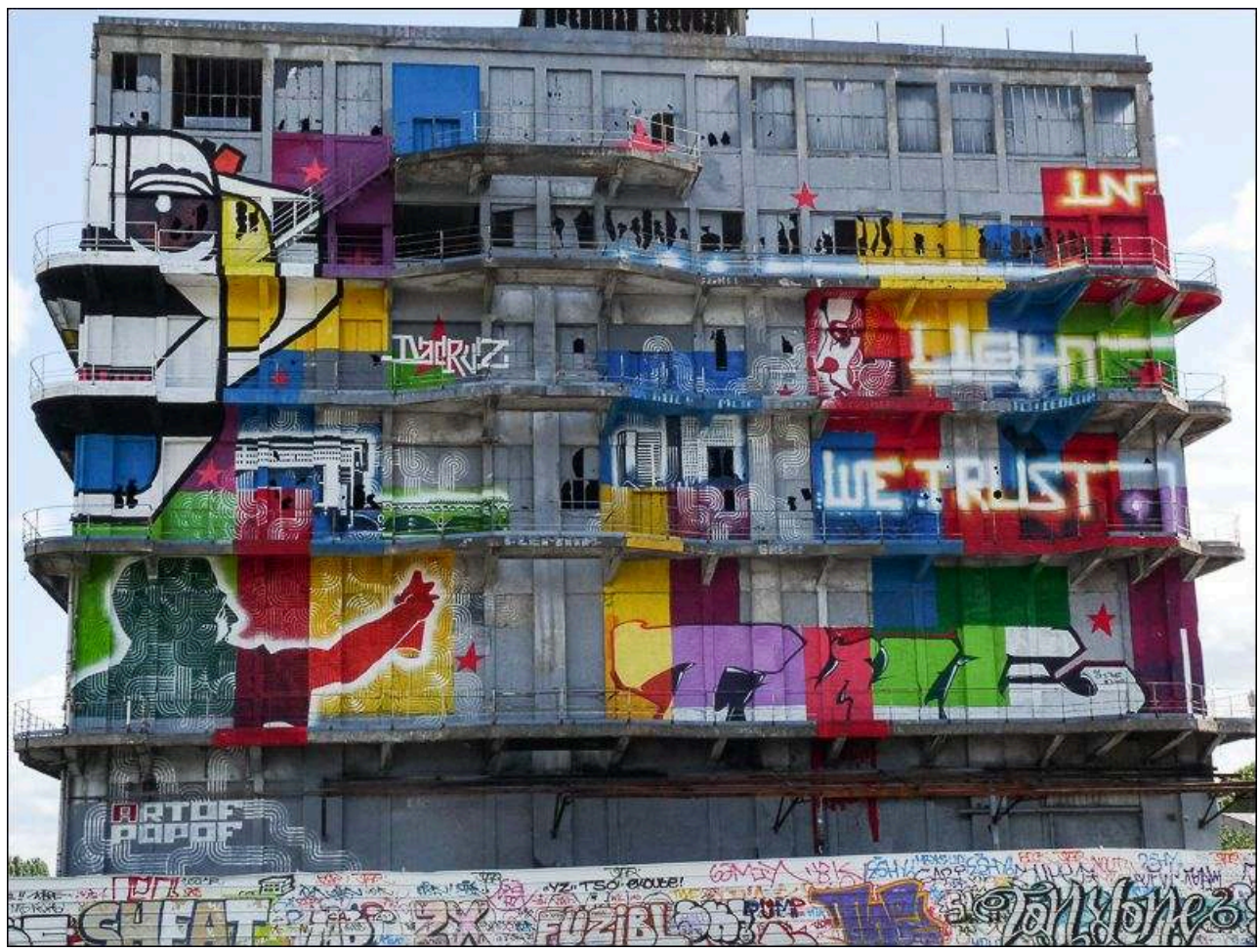

Auteur : C. Ananos municipalité qui collabore avec le CDT dans le cadre de ces projets, de mettre en valeur le territoire dans une stratégie économique centrée sur la culture, le loisir et le tourisme, au travers de son patrimoine industriel mais aussi au travers de pratiques artistiques qui ont su donner d'autres usages au lieu et l'étoffer de valeur esthétique et symbolique par leur appropriation créative.

Cette fois-ci, le lieu off est investi par des artistes issus de pratiques off mais qui s'inscrivent dans un processus in de logiques événementielles touristiques. L'esthétique « graffiti » des Magasins généraux est remaniée sur commande du CDT93, qui demande une unification esthétique des graffitis sur les façades externes, donc visibles. Remarquons que dans une stratégie de marketing territorial qui ne concerne pas que le bâtiment des douanes, le CDT 93 a d'abord proposé au TWE de repeindre le bâtiment des douanes mais le projet n'a pas abouti du fait de différends entre le crew et d'autres acteurs de l'Eté du Canal. Et si le collectif PoDaMa a hérité du projet, c'est du fait de leur implication artistique à l'échelle locale, et de leur proximité géographique avec Pantin, et bien sûr du fil conducteur entre ces territoires que constitue le canal. Ces opérations touristiques ont eu un large écho dans la presse régionale mais aussi nationale. Un tel écho est dû à la mise en pratique du champ de compétences de l'agence publicitaire s'installant dans le lieu. Effectivement, outre le territoire, ces opérations permettent de mettre en scène et en valeur ce nouvel acteur qui devient central tout en ayant trouvé un territoire profitable où s'implanter. Le territoire constitue l'intérêt convergent de la commune et de BETC Paris, qui se dote d'un récit, d'un mythe, d'une "storytelling " dans le langage marketing, par le biais du graffiti et du street art. 
Illustration 7 - Page de garde de Graffiti General par Karim Boukercha en 2014

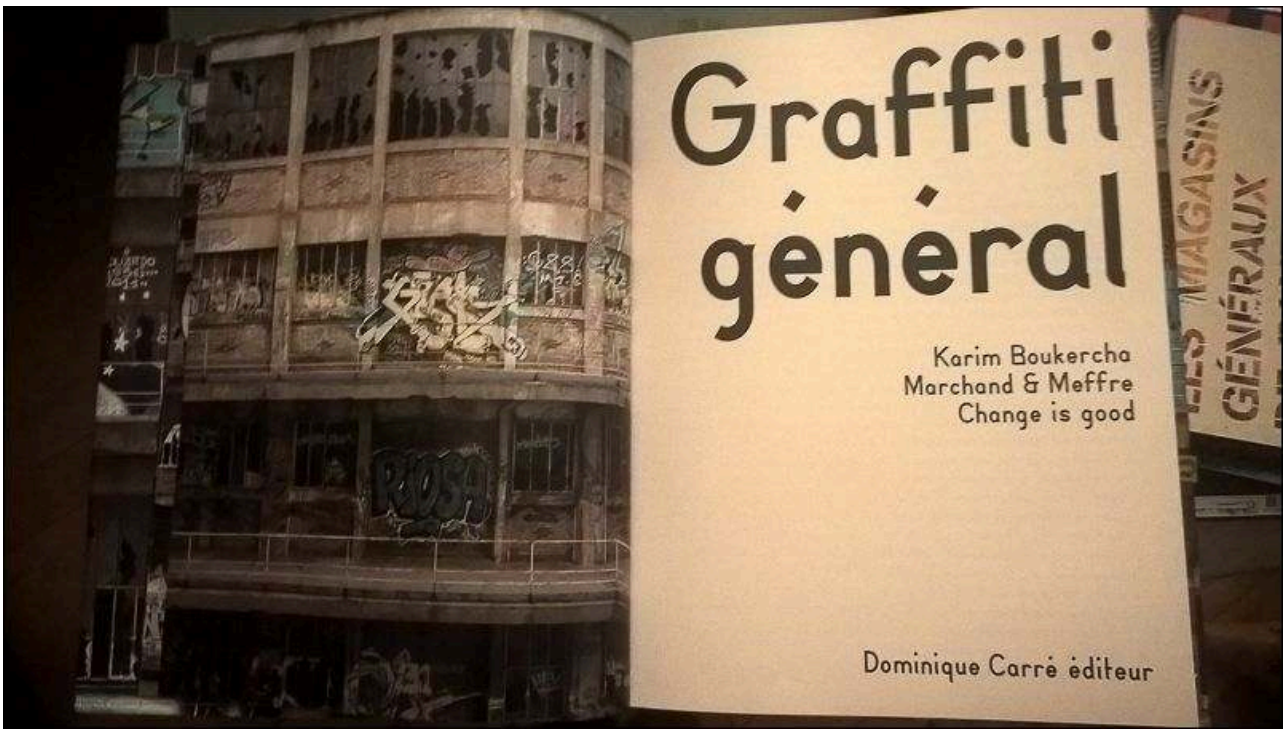

Auteur: C. Ananos.

BETC Paris est une agence publicitaire qui appartient au groupe mondial HAVAS, elle a été créée en 1994 par Eric Tong Cuong, Mercedes Erra et Rémi Babinet. BETC London et BETC Sao Paulo existent en parallèle. Cette agence relève donc d'une stratégie financière à l'échelle mondiale. Initialement installée dans le $10^{\text {ème }}$ arrondissement de Paris, le choix d'arriver à Pantin est délibéré. Les raisons de leur déménagement sont d'abord économiques du fait de la croissance de son activité et de l'augmentation des prix des loyers parisiens. Pantin est une commune du « 9-3» limitrophe de la capitale, ce qui lui donne une situation géopolitique originale. La commune a toujours dû composer avec Paris qui a morcelé et changé le territoire pantinois selon ses besoins. Ce qui historiquement a pu être brutal dans sa relation à Paris peut constituer aujourd'hui pour le territoire un atout : par exemple il y a RER, tramway, métropolitain et routes nationales qui maillent le réseau local de transports; atout qui attire des acteurs économiques aux intérêts financiers. Le bâtiment des douanes entre sa stature industriel et ses graffitis incarnent les deux aspects de la commune, la relation à Paris autant que son appartenance culturelle à la Seine-Saint-Denis. D'une obligation à déménager, l'opération devient alors pour l'agence publicitaire une opportunité de revendiquer une démarche et une identité alternatives se centrant sur les termes et notions de création et d'innovation, et finalement de se créer pour elle-même une identité symbolique.

Pour l'occasion l'agence remodélise son site internet ${ }^{11}$ avec l'opération « Hello Pantin » qui fait la promotion de son installation dans l'Est parisien. Elle crée un autre site dédié aux Magasins généraux ${ }^{12}$. Sur ce site, la souris simule l'effet d'une bombe aérosol), elle " peint » en même temps qu'elle bouge, référence évidente au graffiti. Un autre site internet est créé, sur lequel il est possible de visiter et de tagger virtuellement (avec la souris) le bâtiment ${ }^{13}$. Ce site a pu être développé grâce aux deux photographes engagés par BETC qui ont pris tout le bâtiment en photographie; l'agence possède ainsi une base de données conséquente de graffitis et street art. Les archives photographiques ont servi à la création du site graffiti général, sur lequel on peut visiter et tagger virtuellement le bâtiment, mais sont aujourd'hui la propriété de BETC Paris et donc 
peuvent être réutilisées par l'agence. Elles ont d'ailleurs aussi servi à la publication d'un livre du même titre que le site, Graffiti Général, en 2014. Rémi Babinet en a rédigé la préface dans laquelle il raconte sa « storytelling " entre lui, incarnant alors l'agence, et le bâtiment. Il explique son coup de cœur pour le lieu et ses graffitis et revendique l'installation de BETC Paris dans ce lieu du fait de ce coup de cœur artisticopatrimonial. L'agence inaugure en présence du maire de Pantin le lancement du livre avec une séance de dédicaces de l'auteur, Karim Boukercha, un tirage photographique de certaines images du bâtiment et un buffet, à Artcurial, la maison de ventes aux enchères située sur les Champs Elysées. Il est aussi commandé à Karim Boukercha un inventaire des œuvres que l'on pourrait conserver en les retirant du bâtiment des douanes avant sa rénovation. Cet inventaire a donné lieu à l'extraction des œuvres répertoriées dans l'inventaire. BETC en a fait don à la mairie de Pantin, don qui s'avère être à double tranchant quand on considère les moyens et espaces demandés par le stockage de telles œuvres (principalement des portails et vitraux mais aussi des morceaux de murs), et qui engendre la question de leur devenir et éventuelle accessibilité au public, question encore en débat au sein de la municipalité. Cela permet aussi à BETC de se donner un statut de mécène, au sens soutien à l'art, vis-à-vis de la presse et d'afficher des valeurs altruistes et éthiques où l'art est considéré comme public. Une des clauses des arrangements entre la mairie et BETC pour aboutir à ce don constituait en l'interdiction formelle pour la mairie de Pantin d'en faire la moindre promotion tant que BETC n'était pas formellement installée, seule BETC pouvait en parler à la presse (voir Annexe 2). Notons aussi que finalement, l'agence publicitaire en question ne s'est pas inscrite véritablement dans une démarche de mécène en l'occurrence que ce soit pour les artistes off concernés, ou le graffiti et le street art en soi : ils ne sont pas à l'honneur dans la programmation culturelle actuelle du lieu et aucun des artistes n'a reçu un soutien financier de la part de l'agence. Ce qui factuellement contredit le statut de mécène que BETC Paris s'est donné grâce au graffiti et au street art. Ces éléments mettent en évidence l'importance des jeux d'influence et de communication et les enjeux qui en sont au cœur : l'enjeu de l'agence publicitaire est ici de faire sa propre campagne publicitaire dans une stratégie socio-économique sur le long terme. BETC Paris est aujourd'hui la première agence publicitaire française, ce qui fait d'elle le leader de leur marché. Ces opérations confortent leur position de leader et leur prédominance du marché. Le génie de l'agence réside ici non seulement à conforter ces atouts déjà en main mais aussi à s'attribuer un nouveau rôle dans la production et médiation urbaine et culturelle de Pantin et de la métropole. Ce faisant, cela leur permet de développer de nouveaux champs de compétences de façon à pouvoir s'investir à de nouveaux marchés financiers. En cela les Magasins généraux constituent un cas d'étude original car nous assistons à la transformation d'un lieu off en lieu in dont la transformation même devient une opportunité pour cet acteur privé de s'implanter la géopolitiquement, de s'attribuer de nouvelles compétences et de revendiquer ainsi un rôle de décideur dans les processus de production et médiation urbaines. 


\section{D'un lieu off, spot à graffeurs, à un lieu in, « lieu de création, de production, d'innovation et d'expérimentation »}

\section{Le graffiti et le street art comme outils de valorisation symbolique d'un lieu vacant à un outil de mise en scène d'opérations urbaines}

En s'appropriant les anciennes douanes de Pantin, les graffeurs et street artistes l'ont transformé en lieu de la culture du off autant qu'en lieu off, il est devenu un de leurs spots. Le terme spot est un terme anglais que le français a récupéré justement du fait de sa flexibilité de ce qu'il peut signifier mais aussi car conceptuellement il renvoie à un élément ou une idée qui se démarque dans le paysage, symbolique ou physique. Il est communément utilisé dans les milieux du graffiti et du street art, et désigne en général l'emplacement d'une pièce. Il désigne aussi par extension un lieu propice au graffiti : peu ou pas fréquenté, avec des murs et autres formes urbaines vierges et accessible (une sécurité contournable et une enceinte franchissable). Le graffiti a repris les codes et les techniques de promotion publicitaire puisque l'objectif premier était à l'origine, et est encore bien souvent, la reconnaissance des pairs obtenue par une visibilité optimale du blase, une stratégie publicitaire avec pour message le blase. On désigne aussi une courte vidéo promotionnelle par l'expression spot publicitaire. Tous ces usages du terme spot rendent son usage pertinent dans ce cas, permettant de naviguer aisément entre deux des univers qui se rencontrent, le graffiti et le street art d'un côté face à une agence publicitaire de l'autre, et d'être communément utilisé par leurs acteurs respectifs. Ici le lieu off est un spot car il a tous les critères recherchés par les graffeurs et notamment ceux de terrain; il en devient une sorte de double spot publicitaire : à la faveur du graffiti et du street art d'abord dans une logique off puis dans une logique in à la faveur du territoire pantinois et de l'agence publicitaire qui s'y est installée.

Les acteurs du off ont donné de la valeur symbolique au bâtiment des douanes en lui redonnant des usages en dehors du cadre marchand. Le street art et plus particulièrement le graffiti incarnent une marginalité urbaine qui implique un processus de désobéissance aux règles dictées et parfois un mode de vie nocturne. Or, le système libéral actuel se nourrit des pratiques off car il reconnait dans la liberté de ces pratiques la part de dérégulation supposée permettre l'innovation (Génin, 2016) ; c'est exactement ce que nous voyons se produire dans ce cas précis au travers de la rénovation urbaine de ce lieu.

Ce lieu illustre cette image de ces pratiques urbaines et renforce cette valeur symbolique, car le lieu s'est doté d'une aura par le nombre et la variété des graffitis que l'on peut y trouver. Par le processus de promotion mis en place par BETC, il va devenir emblématique du graffiti alors qu'il n'est pas le seul bâtiment à avoir été propice à l'émergence d'un spot dans ce milieu. Dès les années 1980, le terrain de Stalingrad, anciennement situé 22 boulevard de la Chapelle, visible alors du métro aérien, a été un des premiers terrains français à marquer le milieu du graffiti en tant qu'espace pour peindre et socialiser (par exemple NTM pouvait y être entendu avant de devenir célèbre). Le phénomène n'est donc pas isolé dans le milieu et le bâtiment des douanes n'est pas le seul à avoir été qualifié de "cathédrale " par la presse, par exemple le projet Tour 13 dans le $13^{e}$ arrondissement a aussi été qualifiée de « cathédrale » de l'art 
urbain contemporain par d'autres médias. Le terme cathédrale ici renvoie à la fois à la masse architecturale que constituent les lieux en question mais aussi à une stratégie sous-jacente de monumentaliser les lieux en leur donnant une valeur symbolique de patrimoine bâti et artistique. Dans le cas du $13^{e}$, la stratégie politique de promotion du territoire par le street art est menée par une collaboration avec la galerie parisienne Itinérance (Crapanzano, 2018); et l'exemple de la Tour 13 diffère en cela avec le bâtiment des douanes à Pantin: il part d'une logique in organisée par la galerie Itinérance avec la mairie du $13^{\mathrm{e}}$ arrondissement. Les artistes n'ont pas investi le lieu dans une logique off, ils ont été invités à intervenir. Le grand public, après des heures de queue, pouvait visiter gratuitement l'endroit lors du mois d'octobre 2013.

Le graffiti et le street art en tant qu'outils porteurs de valeurs symbolique du off sont donc pertinents dans des logiques in de marketing territorial et de mise en événement de la ville créative. Si dans le cas du bâtiment des douanes, nous voyons une transition du off au in par l'utilisation du off, précisons que cette utilisation a eu lieu avec l'aval des artistes concernés dans la majeure partie des cas, soit par Karim Boukercha lors de la réalisation de l'ouvrage Graffiti Général, soit par la commune de Pantin lorsque leurs œuvres ont été retenues pour le projet de patrimonialisation lancé par BETC Paris. L'élément clé ici est d'engager Karim Boukercha, acteur du graffiti, donc du off, reconnu par les graffeurs à la fois pour son travail en tant que graffeur et pour son travail de reconstitution historique du graffiti dans le métropolitain parisien contenu dans l'ouvrage Descente interdite (2011). Il a réuni nombre d'archives et recueilli les témoignages des protagonistes du graffiti dans le métropolitain parisien de 1984 à 2011 et peut revendiquer une expertise du graffiti de la région parisienne. Accessoirement, l'agence publicitaire délègue intelligemment un travail de fond, celui de reconstitution historique du graffiti dans les terrains et friches urbaines, mais avec un parti pris sur le bâtiment des douanes, le livre étant une commande, contrairement aux autres ouvrages de Karim Boukercha. Le propos de l'auteur est donc nécessairement différent, bien qu'il ait assuré en réponse à un questionnaire, avoir pu imposer ses conditions, à savoir le respect de l'histoire du milieu, de ses personnages locaux et de ses règles (voir Annexe 3).

L'agence publicitaire s'est ainsi inscrite dans le récit qui se construit autour de la rénovation urbaine de l'Est parisien et dans l'histoire du graffiti et du street art en récupérant la valeur symbolique transmise par les acteurs off au lieu et en se l'appropriant comme caractéristique de l'entreprise, que de cultiver la marge, la créativité artistique et de participer à la production d'événements urbains. Jean-Luc Rigaud, dans l'ouvrage sur les Magasins généraux commandé par la Semip, nous indique d'ailleurs que quelques tags et pièces auraient été préservés et intégrés à la nouvelle esthétique du bâtiment, à l'étage du siège de BETC, en guise de source d'inspiration.

\section{Le lieu in d'aujourd'hui : nouveaux occupants, nouveaux usages, nouveaux événements}


Illustration 8 - La terrasse du Dock B, le restaurant des Magasins généraux, en 2017

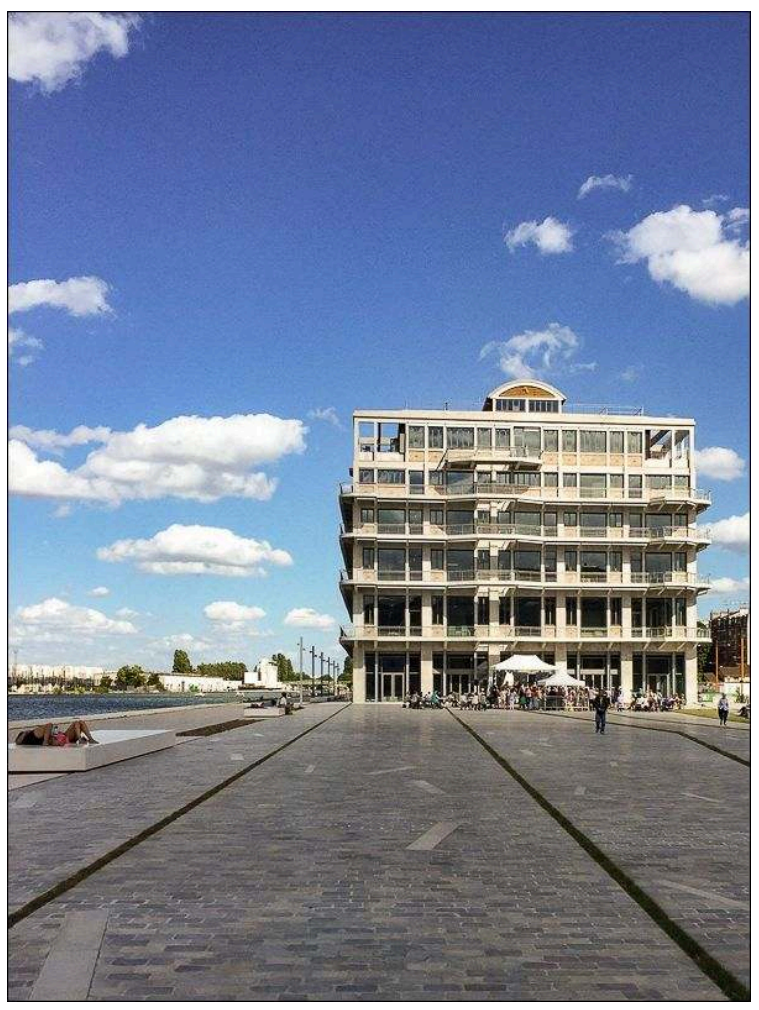

Auteur: C. Ananos

Les Magasins généraux constituent toujours un espace conséquent, à côté d'une place publique, et à proximité d'un square. Locataire du bâtiment, l'agence publicitaire l'a investi en 2016, elle a implanté son siège et gère le lieu dans son ensemble, dont le rezde-chaussée : un espace accessible au public où divers événements sont organisés avec un accueil qu'ils ont appelé « accueil général» mais aussi un restaurant, Le dock B (en référence à l'architecture du bâtiment inspirée des docks londoniens) qui installe sa terrasse sur la place publique avoisinante quand la météo s'y prête. BETC Paris a accueilli dans les Magasins généraux d'autres institutions in qui font du lieu un « hub parisien", selon leurs propres termes, d'activités de logiques événementielles de production culturelle urbaine. Leurs résidents sont Médialab93, « incubateur coopératif de jeunes créatifs de la Seine-Saint-Denis », le centre national Edition Art Image (CNEAI) et le Poste Général, un fabricant de podcasts directement lié à l'agence. Le CNEAI est un professionnel de la culture au sens traditionnel, tandis que Médialab93 illustre les tendances entrepreneuriales actuelles des «jeunes créatifs » en mettant en place une professionnalisation de la mise en lien et en événement tout en revendiquant un attachement au territoire du " 93 ». Le Poste Général est un outil de communication $\mathrm{du}$ lieu et de l'agence. Ces acteurs sont eux-mêmes des outils de légitimation d'implantation du lieu in. L'agence publicitaire, par la présence de ces professionnels de la médiation culturelle et de la production urbaine, délègue une partie de la conceptualisation de la programmation culturelle du lieu et l'insère dans le réseau des centres et lieux culturels parisiens (au sens métropolitain). La stratégie municipale de son côté est de faire de Pantin une série de spots, de sites à voir, du grand Paris; en d'autres termes, de pouvoir maintenir une identité locale tout en mettant à profit sa dépendance socio-économique vis-à-vis de à la capitale. L'agence publicitaire et la 
mairie de Pantin partagent des enjeux communs aux objectifs différents à terme; l'une est centrée sur le succès et le rayonnement de son agence, l'autre de son territoire communal et de ses habitants.

Une série d'événements a eu lieu depuis l'emménagement de BETC Paris, à commencer par l'inauguration des lieux à la suite de la rénovation et du fait de cette installation, en présence des personnalités politiques territoriales mais aussi Claude Bartolone, alors président de l'Assemblée nationale. La programmation culturelle des Magasins généraux, sous la direction de l'agence publicitaire, illustre bien sa stratégie politique dans le domaine culturel. Attardons-nous sur quelques-uns particulièrement parlants.

En avril 2017, dans le cadre du mois de la photo, l'événement les "Grands Parisiens » est lancé en partenariat avec les studios Harcourt. Trois studios sont installés au rezde-chaussée où les habitants de Paris peuvent venir se faire photographier gratuitement (sur réservation) en échange de la cession de leur droit à l'image pour l'exposition de ces mêmes photographies dans le hall du bâtiment. D'après les observations menées sur place pendant deux journées sur deux weekends, l'événement a attiré parisiens et banlieusards, des familles, des jeunes, des couples etc. Le but plus général est de construire une identité territoriale "Grand Paris» dont l'agence publicitaire serait une composante clé; le terme banlieue ou périphérie ou Pantin n'est pas usité dans le cadre de cet événement. La promotion du Grand Paris prévaut. Dans le même esprit, en juillet 2017, la rencontre événement organisée par le Club Ville hybride-Grand Paris a lieu pour échanger et envisager les logiques événementielles de production de la ville avec la perspective de l'arrivée de l'Exposition Universelle et des Jeux Olympiques sur les territoires du Nord-est Parisien des communautés d'agglomération Paris Plaine Commune et Est-Ensemble.

L'espace public des Magasins généraux accueillent aussi des expositions comme celle organisée par le CNEAI, «The house of dust by Allison Knowles ", des concerts, comme celui du groupe TAUR (produit par Pop Records, i.e. BETC Pop ${ }^{14}$ et le label Polydor) ou la Grande Braderie de la Mode, en partenariat avec l'association de lutte contre le sida Aides, qu'ils accueillent désormais annuellement. Ils couvrent donc plusieurs champs et disciplines artistiques et culturelles dans leur programmation, bien qu'aucun ne s'attarde sur le graffiti et le street art qui ont presque disparu du lieu, et complétement de la programmation culturelle.

Cette étude de cas amène à se questionner sur les nouvelles modalités de production et de médiation culturelles de la ville car elle montre l'émergence d'un acteur privé dont le domaine de compétences, la publicité, est régi par la Chambre de Commerce Internationale (CCI) dans ses modalités. Les stratégies des différents protagonistes de ce cas du bâtiment des douanes de Pantin ont permis l'implantation d'un centre culturel qui en soi n'a rien de particulièrement innovant si ce n'est sa direction dévolue à l'agence publicitaire. 
Illustration 9 - Exposition Les Grands Parisiens aux Magasins généraux en 2017

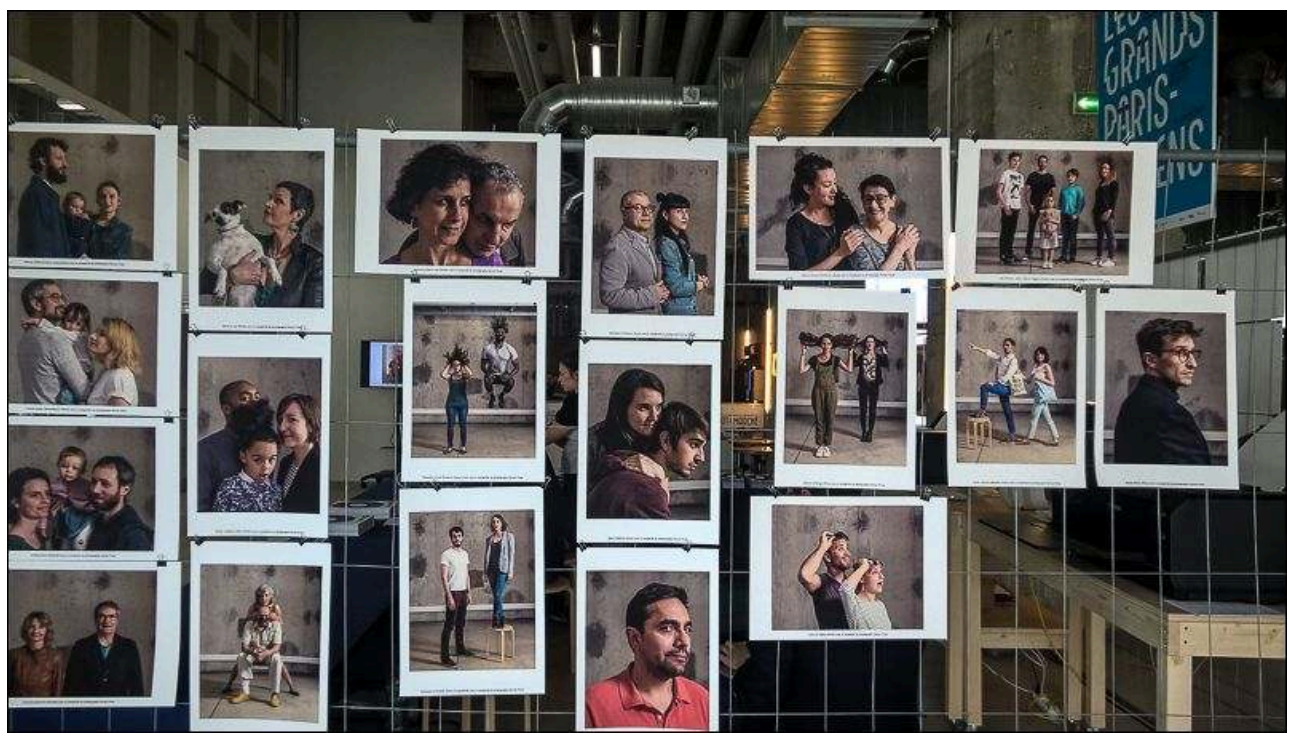

Auteur : C. Ananos

Ces éléments amènent à se questionner à la fois sur les processus hybrides in et off de production urbaine et sur l'émergence de nouveaux opérateurs d'intermédiation urbaine, c'est-à-dire les logiques et les nouveaux opérateurs de production de la ville mises à l'œuvre à Pantin sur l'initiative de BETC Paris, en partenariat avec les pouvoirs publics territoriaux, et sur leurs effets. Dans le contexte de leur déménagement/ emménagement, Mercedes Erra et Rémi Babinet, présidents fondateurs de BETC, reconnaissent opérer «la meilleure campagne de publicité qu'elle pourra faire pour elle-même $»^{15}$ : l'agence s'est approprié de nouvelles compétences sans changer de statut par rapport à leur activité publicitaire en suivant une logique opportuniste. Le bâtiment des douanes a produit une esthétique évoquant celle du squat artistique, qui privilégie les espaces industriels. Cette esthétique est une composante de la mise en scène de lieux temporaires en tant qu'espaces alternatifs et expériences singulières (Pinard, Vivant, 2017). En tant qu'opérateur d'intermédiation urbain, BETC assume les rôles de producteur, organisateur et gestionnaire d'événements culturels urbains : elle peut mettre en avant ainsi les valeurs et projets culturels qui vont dans son sens, auxquels elle adhère, autant qu'être à l'origine d'événements culturels ou de leur mise en scène et de leur patrimonialisation comme cela a été le cas avec le graffiti et le street art. BETC s'inscrit ainsi dans la participation à l'émergence et mise en place de la « ville créative » et une stratégie de redéfinition du territoire par l'activité touristique.

\section{BETC et les Magasins généraux, une stratégie « créative » à l'échelle métropolitaine}

38 Les Magasins généraux en tant que lieu in constituent un révélateur pertinent des acteurs de production et gouvernance urbaines qui se mettent en place alors que le gouvernement français donne de plus en plus d'importance économique et décisionnelle au secteur privé et notamment à des acteurs tels que BETC Paris. Dernièrement, BETC a conçu le site internet promotionnel à but touristique de la France $^{16}$ : sous l'égide du ministère de l'Europe et des Affaires étrangères et en 
collaboration avec d'Atout France, agence de développement touristique, BETC Digitial a refondé le site et a indiqué à son sujet: "[...] France.fr constitue une vitrine du meilleur de l'art de vivre de nos territoires. Il veut illustrer la créativité, la convivialité, la vitalité d'une France continuellement en mouvement, innovante, contemporaine et culturelle " (Nébia, 2018). BETC s'est aussi chargé de la conceptualisation et réalisation d'un guide « culturel » du grand Paris à destination des grands parisiens, intramuros et banlieusards. En parallèle, Rémi Babinet préside désormais le conseil d'administration du Centre National de la Danse à Pantin après sa nomination en décembre 2017 par le président de la République, Emmanuel Macron. Sa collègue Mercedes Erra a présidé le conseil d'administration de la Cité nationale de l'histoire de l'immigration de 2010 à 2012. Elle préside celui du Palais de la Porte dorée depuis 2012. Ces autres institutions à la tête desquelles les présidents-fondateurs de BETC Paris se trouvent sont des établissements publics. Rappelons brièvement que BETC fait partie du groupe mondial HAVAS dont le président directeur général est Yannick Bolloré.

Ces éléments révèlent une volonté politique de la part de l'agence publicitaire de s'inscrire dans les processus de production et d'intermédiation culturelles de l'Est parisien et de la métropole. Il est possible de constater une cohérence entre la stratégie politique gouvernementale de privatisation progressive du service public et cette volonté politique de l'agence par les nominations de Rémi Babinet et Mercedes Erra à la direction d'établissements culturels publics. Aussi, en réduisant les moyens financiers des collectivités territoriales, le gouvernement favorise l'émergence de centres culturels tels que les Magasins généraux.

Le statut et les nouvelles fonctions que BETC prend passe par une approche géostratégique traditionnelle qui est d'abord l'implantation d'un quartier général, d'une base, et donc une implantation territoriale qui permet une action directe sur ledit territoire. Ces quartiers généraux sont le siège de BETC, lieu «de création, de production, d'innovation et d'expérimentation ». Le nouveau lieu in n'a pas d'autres relations directes avec ce qui a été le lieu off que celle de la "culture ", notion assez vaste et avec assez de définitions et conceptualisations au travers de l'histoire et des champs disciplinaires pour que l'on puisse tout et rien y entendre.

41 Les termes de création, production, innovation et expérimentation, que l'agence publicitaire a choisi pour décrire les Magasins généraux après leur réhabilitation font partie d'une stratégie globale de redéfinition même de leur champ de compétences dans les processus de production urbaine mais aussi dans une conceptualisation et fabrication émergentes de la ville, qui sont celles de la «ville créative ». Comme il a été mentionné, les publicitaires sont des acteurs qui s'approprient le champ lexical "créatif » dans leur propre champ de compétences. Ces termes sont utilisés à cet escient; ils ne sont jamais vraiment définis dans le discours public que donne BETC mais ils sont récurrents. Leur présentation «à propos » des Magasins généraux sur le site internet du lieu est particulièrement significative sur la construction d'un discours promoteur des artistes et de la création qui va servir leur activité économique moteur, la publicité d'un client.

Illustration 10 - Éléments de langage extraits du site des Magasins généraux

Un centre de création Grand Parisien fondé par BETC 
Ancien site industriel situé au bord du canal de l'Ourcq à Pantin, les Magasins généraux sont un centre de création, fondé par BETC, qui participe à l'énergie et à l'émergence du Grand Paris. Catalyseur de rencontres entre les créateurs et le public, ils rassemblent plusieurs acteurs de la création qui contribuent tous à la programmation.

Expérimenter de nouveaux modèles pour la création

Convaincus que la culture et la création ont le pouvoir de relier les gens les uns aux autres, les Magasins généraux développent de nouveaux contenus culturels, sans limite de forme - saisons culturelles, festivals, expositions, rencontres, ateliers, concerts, projets d'édition, programmation hors-les-murs, résidences - avec des créateurs de tous horizons : artistes, designers, architectes, musiciens, danseurs, chefs cuisinier...

À travers des collaborations inédites, les Magasins généraux peuvent associer au gré de leurs projets les différents résidents du lieu, des marques, des acteurs locaux et des institutions partenaires.

Une même ambition anime toute la programmation: repousser les frontières ordinaires pour explorer le futur, les idées et les sujets qui agitent la société contemporaine.

Les Magasins généraux sont autant un lieu de vie qu'un lieu d'expérimentation créative et culturelle. Ils s'inscrivent dans l'écosystème déjà bouillonnant du Grand Paris. Notre envie avec ce projet est d'inventer de nouveaux types d'objets culturels qui aient un sens, une utilité, qui soient innovants à la fois dans le fond, les sujets qu'ils adressent, et dans leur forme, toujours unique. Le faire en imaginant de nouvelles collaborations entre les artistes, les institutions, les médias, les marques et le public est notre priorité.

Eugénie Lefebvre, directrice des Magasins généraux

\section{Une création signée BETC}

Avec les Magasins généraux, BETC explore de nouveaux terrains d'expression et de dialogue entre l'art et le commerce, entre la culture et l'innovation, participant à l'émergence de nouveaux formats et nouvelles écritures publicitaires

Cela a toujours fait partie de son ADN : BETC accompagne depuis des années de grandes marques culturelles comme la Philharmonie de Paris, les Ateliers Médicis, le Musée national de l'histoire de l'immigration, la Fondation Galeries Lafayette ou plus récemment, le MAD.

Les Magasins généraux sont ainsi la continuité naturelle d'un engagement historique.

Depuis son origine, BETC s'est développé en créant des stratégies de transformation originales pour ses clients: entreprises, associations, institutions, artistes. La création comme force de transformation a toujours été son obsession. Avec les Magasins généraux, BETC, dans un monde des médias, de la culture, et de la communication en plein bouleversement, continue à défricher de nouveaux territoires et à expérimenter de nouveaux formats.

Rémi Babinet, président-fondateur de BETC et des Magasins généraux

Source : https://Magasinsgeneraux.com/fr/a-propos consulté le 23/05/2018.

Dans cette présentation, il y a une liste de formes urbaines et artistiques déjà existantes et développées dans les centres et événements culturels et véritablement la revendication de toutes les utiliser en fonction des intérêts des projets respectifs, 
quoique toujours au bénéfice de l'agence publicitaire et de ses résidents. Les événements décrits précédemment qui se déroulent dans le nouveau lieu in illustrent le décalage entre le discours donné et les faits. Les champs lexicaux utilisés s'attachent à lier "art et commerce", et transforment les institutions culturelles en " grandes marques ", ce qui est révélateur d'un mode de pensée adapté au néo-libéralisme. L'appropriation entière du lieu «fondé par BETC » écarte l'action des pouvoirs publics territoriaux (ici la mairie de Pantin et le CDT 93) qui facilitent pourtant très largement l'implantation de BETC et qui ont déjà enclenché territorialement le processus d'institutionnalisation et de patrimonialisation du graffiti et du street art. Elle illustre l'implication de BETC dans le processus de transformation de Pantin et de l'Est parisien en «ville créative », et la récupération de cette compétence à la transformation urbaine par l'art.

La notion de ville créative connaît un certain succès dans la communauté scientifique, mais aussi chez les acteurs et opérateurs urbains. Elle peut être comprise comme un projet politique libéral (Vivant, 2009) au sens états-unien, le projet de villes qui prônent plus de tolérance concernant les mœurs et les choix de vie. Pour autant, selon les constats et analyses actuelles, ce projet s'accompagne souvent, et c'est visible à Pantin aussi, d'un processus de gentrification. BETC Paris, par son statut et celui de ses salariés correspond au schéma de populations qualifiables de créatives, et vise ses pairs à travers les partenaires et projets culturels. Se pose alors la question de savoir si nous souhaitons de tels acteurs à la tête des processus de fabrication de nos villes.

Illustration 11 - Image d'arrière-plan de la page d'accueil du site des Magasins généraux

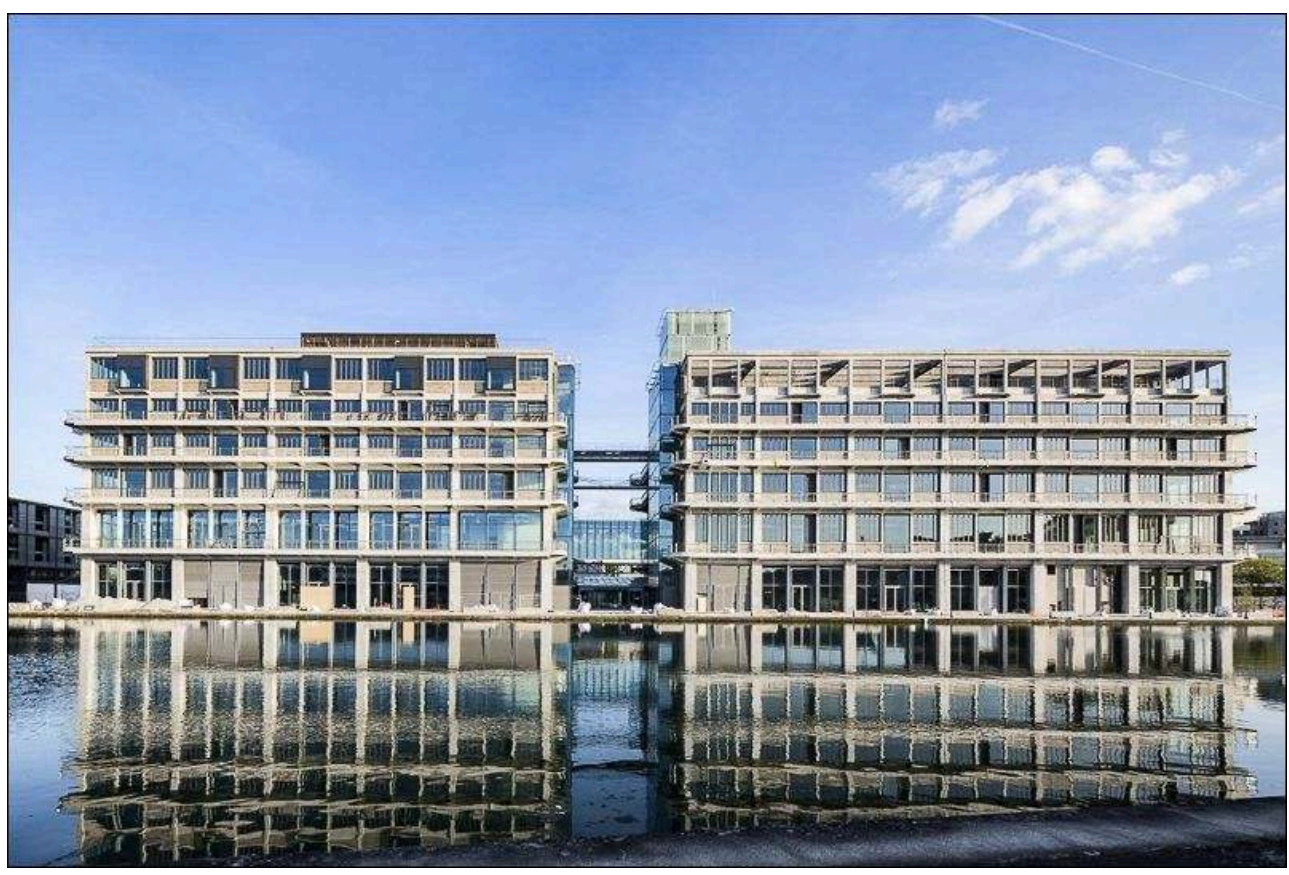

Auteur : BETC, 2017.

\section{Conclusion}

Par l'étude de ce cas particulier, nous avons pu constater l'appropriation d'une friche urbaine par des graffeurs et des street artistes qui en ont alors fait un lieu de la culture 
du off autant qu'un lieu off en soi. Ce lieu off a alors été mis en événement de nouveau par le biais du graffiti et du street art avec le collectif PoDaMa dans une logique événementielle à buts touristique et marchand mais aussi patrimonial et publicitaire. On assiste à un processus d'hybridation du in et du off de production de la ville lors de la réhabilitation des Magasins généraux. Cette mise en événement et réappropriation in du off aboutit à la création d'un lieu in légitimé par et inspiré du off dont le gestionnaire est BETC Paris, une agence publicitaire qui se positionne dans le rôle d'opérateur d'intermédiation culturelle de la production urbaine à partir d'un ancrage territoriale stratégique.

Le graffiti et le street art sont à la mode depuis assez longtemps pour ne pas être qu'un effet de mode, ils se pratiquent désormais légalement et illégalement, et en viennent à s'inscrire dans les modalités de production urbaine in; ils deviennent alors soit littéralement un événement (performances) soit une mise en événement de son esthétique et de ses lieux. Dans le cas présent l'occupation temporaire du lieu n'a jamais été envisagée comme pérenne, mais a été utilisée devenant un outil de mise en scène de la réhabilitation du bâtiment au profit du territoire et de la visibilité socioéconomique de BETC Paris. Ce faisant, la transformation de milieux urbains par l'art est intégrée à une conception mercantile de la culture et de l'art qui coïncide avec une stratégie de développement touristique porté par les pouvoirs territoriaux de la SeineSaint-Denis, en l'occurrence autant la Ville de Pantin que le comité départemental du département.

L'innovation, la création, l'expérimentation, ce sont aujourd'hui des termes utilisés pour faire décrire et faire la promotion de nouvelles modalités hybrides de production urbaine mais à quel profit et pour quels effets? Cette hybridation des processus in et off de production urbaine de la ville questionne effectivement la place grandissante des usages marchands au sein des occupations temporaires et du nouveau modèle socioéconomique urbain qu'elles impliquent, celui de la ville créative. 


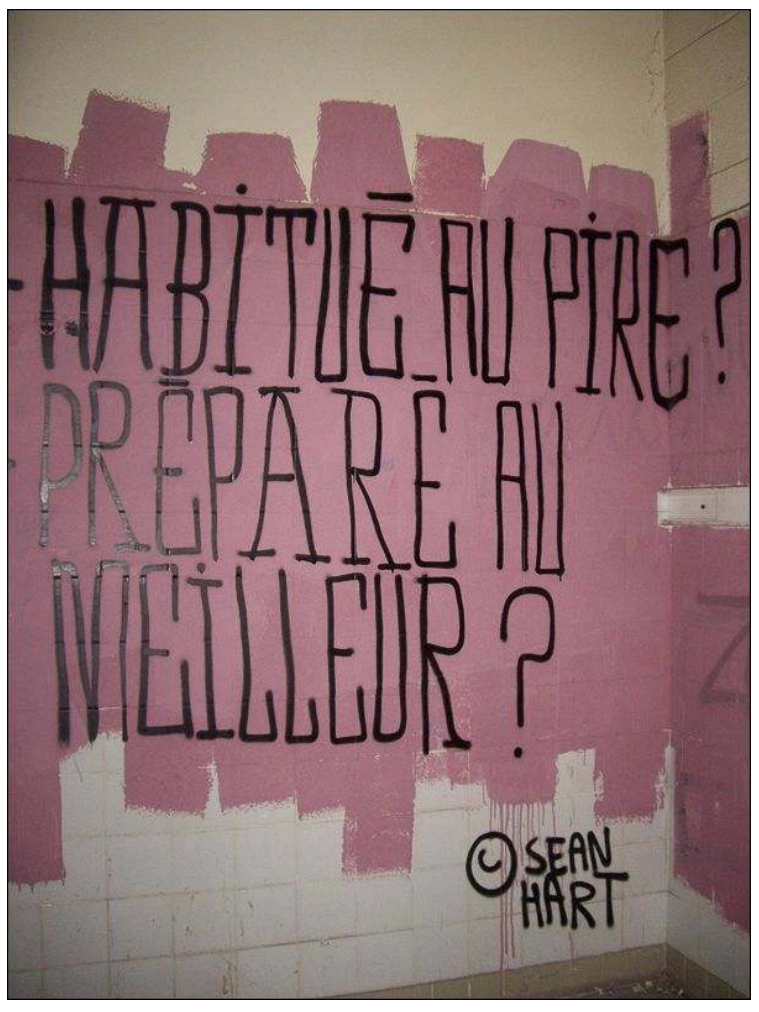

Auteur : C. Ananos

\section{BIBLIOGRAPHIE}

Abittan D., 2016. BETC, l'agence de pub branchée qui faisait travailler ses salariés dans des bureaux pas si cool, Slate [En Ligne], 27/09/2017. http://www.slate.fr/story/123939/betc-pantinbureaux-libres

A Nous Paris [site internet]. https://www.anousparis.fr/lieu/Magasins-generaux/

Bellanger E., 2001. Pantin, mémoire de ville, mémoires de communaux (XIXe et XXe siècles). Pantin, Ed. Archives municipales de Pantin, 2001.

Belmont S., 2014. Un musée au fil de l'eau. Le Parisien, vendredi 8 août 2014, p. 8.

BETC Paris [Profil facebook]. https://www.facebook.com/BETCParis/ - [Profil Instagram] https:// www.instagram.com/betcparis/ - [site internet]. https://betc.com/fr/

Blanchard S., 2017. Street art, rénovation urbaine et gentrification dans le nord-est parisien : entre marketing urbain et gendermainstreaming. Urbanités, $\mathrm{n}^{\circ} 9,15$ p, http://www.revueurbanites.fr/9-street-art-renovation-urbaine-et-gentrification-dans-le-nord-est-parisien-entremarketing-urbain-et-gender-mainstreaming/

Blog All City [site internet]. www.allcityblog.fr 
Boukercha K., 2014. Graffiti Général, préface de Rémi Babinet. Paris, Dominique Carré éditeur, $224 \mathrm{p}$.

Brucker S., Vechionne M.-A., 2004. Writers : 1983-2003, 20 ans de graffiti à Paris. Résistance Films, 93 minutes.

Cabrera E., 2013. Street art : à Pantin, une cathédrale du graff va disparaître. Rue 89, 14 septembre 2013.

CDT 93 [Profil facebook]. https://www.facebook.com/seinesaintdenis.tourisme/ - [Site internet]. https://www.tourisme93.com/

Crapanzano F., 2018. Street art et graffiti : l'invasion des sphères publiques et privées par l'art urbain. Paris, L'Harmattan, $158 \mathrm{p}$.

Clerval A., Fleury A., 2009. Politiques urbaines et gentrification, une analyse critique à partir du cas de Paris. L'Espace politique [En ligne], 8, 2009-2. https://journals.openedition.org/ espacepolitique/1314

De Aquino P., 2014. BETC brassera le monde à Pantin. Canal le journal de Pantin, n²25, p. 22-30.

De La Brosse J., 2013. Comment Pantin la rouge a séduit le monde du luxe. L'Express, 4 juillet 2013.

Fainsilber D., Silbert N., 2015. A Pantin souffle le nouvel esprit du canal. Les Echos, le 06/03/2015. [En ligne] http://www.lesechos.fr/enjeux/business-stories/globalisation/0204186651226-apantin-souffle-le-nouvel-esprit-canal-1099547.php

Fat cap "Street art and Graffiti" [Site internet] http://www.fatcap.com/

Gatineau P., 2018. Lancement du premier guide culturel du Grand Paris. Journal du Grand Paris [En ligne], mis en ligne le 30/01/2018. https://www.lejournaldugrandparis.fr

Génin C., 2016. Le street art au tournant. De la révolte aux enchères. Paris, Les Impressions Nouvelles, 272 p. (réédition de : Le street art au tournant. Reconnaissance d'un genre, 2013).

Guinard P., 2014. Johannesburg. L'art d'inventer une ville. Rennes, Presses universitaires de Rennes, 326 p.

Gré C., 2014. Street art et droit d'auteur, A qui appartiennent les œuvres de la rue ? Paris, L'Harmattan, $80 \mathrm{p}$.

Harvey D., 2011. Le capitalisme contre le droit à la ville. Paris, Éditions Amsterdam, 93 p.

Kokkoref, M., 1988. Des graffitis dans la ville. Quaderni, n 6, p. 85-90.

Lebeau B., 2014. Une « banlieue créative » dans le Grand Paris ? EchoGéo [En ligne], 27. http:// journals.openedition.org/echogeo/13718

Les Magasins généraux [Profil Facebook] https://www.facebook.com/MAGGENERAUX/ - [Profil Instagram]. https://www.instagram.com/maggeneraux/ - [sites internet]. http://

Magasinsgeneraux.com/fr/ et www.graffitigeneral.com

Mailer N., Naar J., 1974/2009, The Faith of graffiti. Paris, éditions L'œil d'Horus, 128 p.

MédiaLab 93 [Site internet]. https://www.lemedialab93.com/

Nébia A., 2018. Lancement du site France.fr par BETC Digital. CB News, mis en ligne le 14/01/2018. http://www.cbnews.fr/marques/lancement-du-site-francefr-par-betc-digital-a1039856

Pinard J., Vivant E., 2017. La mise en événement de l'occupation temporaire. Quand les lieux artistiques off inspirent les opérateurs in de la production urbaine. L'Observatoire, $2, \mathrm{n}^{\circ} 50$, p. 29-32. 
Rigaud, J-L., 2015. Les Magasins généraux de Pantin, histoires de mutations. Lyon, éditions Lieux-dits, $168 \mathrm{p}$.

Sarrot A., 2012. En Seine-Saint-Denis, le paradis des graffeurs. Metro, 12 avril 2012, p. 13.

Sean Hart [Site internet]. https://seanhart.org/

Ville de Pantin [Profil Facebook]. https://www.facebook.com/VilleDePantin/ - [Site internet]. http://www.ville-pantin.fr

Vaslin, 2017, Esthétique propre. La mise en administration des graffitis à Paris de 1977 à 2017, Lyon, Université Lyon 2 Lumière, Sciencespo Lyon, 662 p.

Vivant E., 2007. Les événements off : de la résistance à la mise en scène de la ville créative. Géocarrefour, 82, 3, p. 131-140.

Vivant E., 2009. Qu'est-ce que la ville créative ? Paris, PUF, 96 p.

Zambeaux E., 2017. Quand l'agence BETC s'implante en Seine-Saint-Denis. France Inter, émission Périphéries, 16/06/2017.

\section{NOTES}

1. Le canal de l'Ourcq traverse le quartier de la Villette juste avant d'entrer sur le territoire pantinois.

2. Pour cette étude de cas : entretiens semi-directifs avec le directeur général des services de la mairie de Pantin, Jean-Louis Heno ; avec le directeur général adjoint du département citoyenneté et développement de la personne (incluant le département de la culture et celui de la prévention publique i.e. la police municipale), Alain Ananos; avec la directrice du pôle Mémoire et Patrimoine, Geneviève Michel ; le directeur du Comité Départemental de Tourisme 93, Daniel Orantin; et avec le directeur du département de la culture, Claude Lechat- tous menés sur les années 2011/2012.

3. Rattachement du Fort d'Aubervilliers à la ville éponyme au milieu du $19^{\mathrm{e}}$ siècle qui isole le quartier des Courtillières au nord de Pantin ; scission des communes des Lilas (1867) et du PréSaint-Gervais (dès 1790), implantation du cimetière parisien (1886) ; tendance sécessionniste du quartier industriel, alors riche, des Quatre-chemins à la fin du $19^{\mathrm{e}}$ siècle ; implantation des divers réseaux de transports (routiers avec les routes nationales RN2 et RN3), percement du canal, qui scinde la commune en son centre, et installation du réseau ferré.

4. Throw-up/ Throwies en anglais, le fait de réaliser un lettrage en «bubble letters» (lettres rondes), souvent couleurs chrome et noir, ou chrome et autre couleur. On les distingue par leur lisibilité, on les retrouve souvent le long des autoroutes, voies ferrées et dans la rue car ils doivent pouvoir se réaliser assez rapidement.

5. De l'anglais piece, tiré de masterpiece, que je traduis par pièce car tous les graffeurs ne revendiquent pas une dimension artistique claire, ou œuvre selon le contexte dans lequel l'ouvrage en question été produit. Elle se doit d'être plus travaillée et va donc être jugée autant sur ses qualités techniques (maîtrise du remplissage, des contours, des coulures, des dégradés etc.) que sur son emplacement.

6. Crew : groupe, équipe. Il est souvent traduit par collectif en français; mais les graffeurs emploient ce terme alors que d'autres acteurs choisissent volontairement le terme collectif, je choisis d'être fidèle à leur terminologie.

7. Horfé commence le graffiti en 1998, il a beaucoup peint en région parisienne et est aussi actif en galerie que dans la rue. Gorey commence à peindre au début des années 1990, d'abord dans la rue, aussi en région parisienne, puis dans les lieux abandonnés. Il est aujourd'hui installé à New- 
York où il a acquis une certaine reconnaissance dans le milieu de l'illustration. Fléo est originaire de Montréal, il commence aussi le graffiti en 1998 et est désormais installé à Paris, où il se consacre désormais à sa pratique artistique.

8. Arone est Itvan Kebadian, il a donc une pratique artistique à la fois qui répond aux codes du graffiti lorsqu'il effectue des lettrages «TWE » et une pratique figurative qui s'assimile au street art techniquement. Il illustre bien la porosité des deux milieux que sont le graffiti et le street art. Il utilise son blase Arone pour du réaliser des pièces de graffiti plus traditionnelles, et son nom pour ses réalisations figuratives.

9. En plus des graffeurs et street artistes, le bâtiment a été ensuite squatté par des populations sans domicile fixe issues de l'immigration, qui l'ont investi de façon permanente. Par suite de l'installation de squats sociaux, la municipalité a renforcé la sécurité du site en y postant un vigile en permanence et en appliquant une politique d'intervention systématique de la police municipale. Auparavant il n'était gardé que par vidéosurveillance et le coût d'une sécurisation accrue ne valait pas la peine face aux graffeurs qui investissaient les lieux temporairement bien que répétitivement. Source: entretien avec Alain Ananos, DGA de la mairie de Pantin, le 30/07/2015

10. Les publicitaires sont parmi les professionnels qui se sont appropriés les statuts et conditions de travail d'artistes (Vivant, 2009).

11. URL : https://betc.com/fr/

12. URL : https://Magasinsgeneraux.com/fr/

13. URL : http://www.graffitigeneral.com/

14. Branche de BETC Paris qui cherche des talents émergents dans la musique, la gastronomie, la mode etc. à promouvoir

15. Citation tirée de l'article de David Abittan, «BETC, l'agence de pub branchée qui faisait travailler ses salariés dans des bureaux pas si cool », publié le 27/07/2016 sur le site slate.fr

16. URL : https://www.france.fr/fr

\section{RÉSUMÉS}

Cet article prend comme cas d'étude la réhabilitation des Magasins généraux à Pantin, ville limitrophe à la capitale française et appartenant au département de la Seine-Saint-Denis (93). Il s'attarde sur le processus de transformation urbaine du bâtiment, de son état de friche à celui de spot du graffiti et du street art pour devenir ensuite un centre culturel géré par l'agence publicitaire BETC Paris. Lors de ce processus de transformation, le graffiti et le street art sont mobilisés comme des outils de valorisation symbolique du territoire. Ce faisant, ils permettent aussi de légitimer la création du lieu institutionnel et la mise en place à sa tête d'un acteur original qui s'inscrit ainsi dans les processus de production et de médiation culturelles et urbaines à l'échelle métropolitaine. En utilisant l'art comme outil de développement économique, l'agence publicitaire aussi bien que les acteurs territoriaux publics favorise l'émergence du modèle socio-économique de la ville créative.

This paper focuses on a study case of the renovation of an industrial building, the Magasins généraux in Pantin, a suburban city bordering the French capital and belonging to the SeineSaint-Denis department (93). Due to the deindustrialisation process, the industrial activites in the building were shut down in 2001 and it became an industrial wasteland. From 2004, it was 
invested by graffiti and street artists to then be transformed in what it is today : a cultural centre managed by the advertising company BETC Paris. During the transformation, graffiti and street art were used as tools both to promote the territory and the agency settling in the building. By their presence in and invasion of the building, they gave it a symbolical value. Such value has been turned into a strategic mercantile one to legitimate the implantation of this original actor on the metropolis scale and to promote a socio-economic model based on the concept of the creative city.

\section{INDEX}

Keywords : contemporary urban art, urban renovation, tourism, Parisian metropolis, advertising agency

Mots-clés : art urbain contemporain, rénovation urbaine, tourisme, agence publicitaire, Pantin

\section{AUTEUR}

\section{CONSTANCE ANANOS}

Constance Ananos, ananosc@gmail.com, est doctorante en anthropologie urbaine à l'université de Paris 8 et rattachée au Laboratoire LAVUE. 